Revue de droit comparé du travail et de la sécurité sociale

3 | 2019

Les migrations internationales de travail

\title{
La protection des droits fondamentaux des fonctionnaires
}

Sergio Gamonal C.

\section{(2) OpenEdition}

1 Journals

Édition électronique

URL : https://journals.openedition.org/rdctss/1509

DOI : 10.4000/rdctss. 1509

ISSN : 2262-9815

Éditeur

Centre de droit comparé du travail et de la sécurité sociale

Édition imprimée

Date de publication : 1 novembre 2019

Pagination : 160-163

ISSN : 2117-4350

\section{Référence électronique}

Sergio Gamonal C., "La protection des droits fondamentaux des fonctionnaires », Revue de droit comparé du travail et de la sécurité sociale [En ligne], 3 | 2019, mis en ligne le 01 novembre 2021, consulté le 13 novembre 2021. URL : http://journals.openedition.org/rdctss/1509 ; DOI : https:// doi.org/10.4000/rdctss. 1509

\section{(c) (†) $९$}

Revue de droit comparé du travail et de la sécurité sociale est mise à disposition selon les termes de la Licence Creative Commons Attribution - Pas d'Utilisation Commerciale - Pas de Modification 4.0 International. 


\title{
SERGIO GAMONAL C.
}

\author{
Université Adolfo IBÁÑeZ, FACUlté de dROIT
}

\section{LA PROTECTION DES DROITS FONDAMENTAUX DES FONCTIONNAIRES}

La Cour suprême et la Cour constitutionnelle du Chili divergent sur la question de la protection des droits fondamentaux des fonctionnaires ou agents du secteur public.

\section{I - LE DROIT}

Depuis la réforme sur les procédures d'emploi de 2006, la loi chilienne dispose d'une procédure spéciale pour la protection des droits fondamentaux des travailleurs ${ }^{1}$. Cette procédure a permis de protéger les droits fondamentaux les plus précieux des travailleurs contre les pouvoirs de l'employeur, par exemple l'intégrité psychologique du travailleur, sa vie privée, son honneur, l'accès au juge, le droit à la non-discrimination, etc ${ }^{2}$. Toutefois, cette procédure ne s'applique en principe qu'au secteur privé, non aux fonctionnaires et agents du secteur public ${ }^{3}$, ce qui a fait l'objet d'un large débat parmi les experts en droit du travail qui estiment légitime d'octroyer une protection similaire aux fonctionnaires ${ }^{4}$. L'essentiel du problème juridique consiste à déterminer si la procédure de protection est applicable au secteur public, conformément au paragraphe 3 de l'article premier du Code du travail qui prévoit la possibilité de combler les vides juridiques des autres statuts de travail (comme celui du secteur public), à condition que cela ne soit pas contraire à ces statuts particuliers. Le champ d'application du Code du travail est examiné en tant que norme de droit commun du travail, applicable également aux fonctionnaires du secteur public en cas de vide juridique.

1 S. Gamonal C., « Droits fondamentaux et protection juridique du travailleur en droit chilien », Revue de Droit du Travail, $n^{\circ}$ 7/8 juillet-août 2010, Dalloz, p. 469.

2 J. L. Ugarte Cataldo, Derechos Fundamentales, Tutela y Trabajo, 2018, Santiago, Thomson Reuters; S. Gamonal C., La Eficacia Diagonal u Oblicua y los Estándares de Conducta en el Derecho del Trabajo, Santiago, 2015, Thomson Reuters.

3 Au Chili, les agents du secteur public et les fonctionnaires disposent de leur propre statut spécial du travail, conformément à la loi $n^{\circ} 18.834$. Dans le cas des municipalités, il s'agit de la loi $n^{\circ} 18.883$. Aucun de ces statuts ne prévoit de procédure judiciaire en matière de défense des droits fondamentaux des fonctionnaires.

4 K. Varas Marchant, «El Código del Trabajo y la función pública: Análisis de la aplicación supletoria de la acción de la acción de tutela de derechos fundamentales", Revista de Derecho Laboral y Seguridad Social, Thomson Reuters Chile, vol. 1, n 3, 2013, p. 43; D. Marzi Muñoz, "Ministerio Público y Tutela de Derecho Fundamentales. La laboralización del sector público en clave de precarización y el inesperado reequilibrio de poder vía ejercicio de derechos fundamentales ", Revista de Derecho Laboral y Seguridad Social, Thomson Reuters Chile, vol. 1, n² 1, 2013, p. 441 ; K. Varas Marchant, «Las vías de protección ante lesiones de Derechos Fundamentales de Funcionarios Públicos", Revista de Derecho Laboral y Seguridad Social, Thomson Reuters Chile, vol. 2, n 1, 2014, p. 232; R. Pereira Lagos, « Competencia de los Tribunales del Trabajo en Vulneración Derechos Fundamentales de Funcionarios Públicos ", Revista de Derecho Laboral y Seguridad Social, Thomson Reuters Chile, vol.1, n² 4, 2013, p. 129. 


\section{II - LA THÈSE DE LA COUR SUPRÊME}

Si la procédure de protection a été initialement envisagée pour le secteur privé, la Chambre du travail de la Cour suprême a statué en faveur d'une unification de la jurisprudence et a ainsi déclaré le bien-fondé de l'application de la procédure aux fonctionnaires ou agents du secteur public ${ }^{5}$.

Dans son arrêt du 30 avril 2014, la Cour énonce qu'il s'agit d'une " procédure nouvelle et spéciale, introduite par la loi 20.087, dans le but spécifique de protéger les droits fondamentaux des travailleurs ". II ne s'agit pas d'une procédure spéciale à proprement parler, mais d'un mécanisme ou d'un ensemble de règles qui permettent au travailleur de faire valoir la protection juridictionnelle de ses droits fondamentaux dans le domaine des relations de travail, dès lorsqu'il les considère bafoués par l'employeur. Cette nouvelle modalité apparaît comme l'aboutissement d'un processus visant à introduire des règles de fond qui explicitent et/ou renforcent les droits fondamentaux des travailleurs, tels que les droits liés à l'interdiction de la discrimination (article 2 du Code du travail) et à la notion de citoyenneté au travail dans l'entreprise (article 5 du même Code). Dans ce contexte et dans le souci de l'exercice effectif des droits fondamentaux des travailleurs, les règles de protection récemment instaurées comblent un vide juridique en établissant une action spécifique pour les protéger, ouvrant ainsi la voie à ce que l'on appelle «l'effet horizontal » de ce type de droits.

La Cour suprême a déclaré que la procédure de protection s'applique pleinement aux fonctionnaires, étant donné que le statut administratif du secteur public ne prévoit pas de procédure similaire à celle du Code du travail, qui permet d'examiner et de résoudre des plaintes pour violation des droits fondamentaux des fonctionnaires dans le domaine des relations de travail. L'arrêt précise que le statut administratif ne contient pas de règle contraire à la protection des droits fondamentaux des fonctionnaires, ce qui exclut que les règles protégeant ces droits puissent être incompatibles avec les dispositions du statut spécial des agents publics. On peut en effet considérer que l'État, en tant qu'employeur, doit garantir le respect des droits fondamentaux des fonctionnaires travaillant sous son autorité.

La Cour précise que le terme "travailleur» utilisé à l'article 485 du Code du travail (relatif à la procédure de protection) doit être compris au sens large et englobe tous les types de salariés qui travaillent aussi bien dans le secteur privé que public, et ont un lien de subordination avec un employeur. À ce titre, elle analyse le contenu de l'article premier du Code du travail, en précisant que les fonctionnaires sont incontestablement considérés - eux aussi - comme des travailleurs.

Ainsi, la Cour renforce ses arguments en affirmant qu'une fois la relation entre le fonctionnaire et l'État comprise comme une relation de travail, bien que soumise à un statut spécial, il ne convient pas de priver l'agent public d'une procédure destinée à déterminer le respect et la validité des droits fondamentaux dans la relation de travail, au seul motif que les normes précitées associent le terme employeur à un contrat de travail - et non à un décret de nomination - ou qualifient l'employeur de gestionnaire ou d'administrateur, omettant ainsi que l'État, dans ses rapports avec les fonctionnaires qui travaillent dans l'administration, exerce des fonctions habituelles de gestion comme tout employeur, ce qui n'est pas incompatible avec le fait qu'il s'agit de fonction publique.

Tout aussi catégoriquement, l'arrêt conclut qu'il n'existe aucune raison juridique valable d'exclure une catégorie de travailleurs - en l'occurrence les fonctionnaires - de la protection

5 Rol 10.972-2013, 30 avril 2014. 
des droits fondamentaux, notamment si l'on considère que la subordination et la dépendance propres aux relations de travail sont également présentes dans le cadre des relations entre I'État et ses travailleurs, cadre dans lequel l'exercice effectif des droits fondamentaux peut être bafoué en raison des pouvoirs exercés par l'État employeur.

Cet arrêt est d'autant plus pertinent qu'il précise que le Code du travail établit un statut de droit commun et est supplétif pour ce qui est du travail des fonctionnaires dans le pays. Stricto sensu, lorsque l'État viole les droits de ses travailleurs (fonctionnaires), il s'agit d'un problème d'effet vertical plutôt qu'horizontal des droits fondamentaux ${ }^{6}$, ce qui rend encore plus impérieuse la protection des plus faibles dans ce domaine.

\section{III - LA THÈSE DE LA COUR CONSTITUTIONNELLE}

Dans un arrêt rendu le 6 décembre 2018, la Cour constitutionnelle a soutenu une autre vision du problème ${ }^{7}$. La décision a été adoptée à la majorité, par cinq Ministres sur neuf.

Un fonctionnaire municipal avait saisi la Cour constitutionnelle d'un recours en inapplicabilité pour inconstitutionnalité. II s'agit d'un recours dans le cadre d'un procès ordinaire où l'une des parties saisit la Cour constitutionnelle au motif - dans ce cas particulier - de l'application d'une règle juridique jugée anticonstitutionnelle ${ }^{8}$. La déclaration d'inconstitutionnalité est particulière dans la mesure où elle concerne l'affaire qui fait l'objet du recours. La norme juridique reste en vigueur et peut être appliquée dans d'autres procès ou affaires. La Cour constitutionnelle a jugé que la procédure de protection des droits fondamentaux ne s'applique pas aux fonctionnaires et agents du secteur public au Chili. Selon elle, avec l'interprétation de la Cour suprême, la compétence des tribunaux du travail est étendue aux questions de contentieux administratif des agents publics, pour lesquels le tribunal civil n'est pas compétent. Or seule la loi peut expressément étendre la compétence des tribunaux, non une simple interprétation de la loi.

La Cour constitutionnelle précise que le paragraphe 3 supplétif de l'article premier du Code du travail est issu du décret-loi n² 2.200 de 1978. À l'époque, on ne savait pas très bien quel serait le régime de travail des fonctionnaires, le Code du travail ou un régime spécial. Le régime de Pinochet avait finalement opté pour un statut spécial de droit public; dans ce contexte, la règle supplétive a perdu son sens et ne devrait donc pas être appliquée.

II est rappelé que lorsqu'un fonctionnaire estime ses droits fondamentaux bafoués, il peut s'adresser, au sein de la même administration, au Bureau du Contrôleur général de la République, entité publique autonome qui contrôle la légalité des actes de l'administration.

La Cour constitutionnelle conclut en ces termes: "Même si le principe selon lequel les agents de l'État régis par le statut administratif sont effectivement soumis à la règle supplétive du Code du travail, en tout état de cause, la conclusion selon laquelle leur protection relève des tribunaux du travail n'est ni logique, ni déterminante. Ainsi, l'application généralisée du Code du travail, en vertu du paragraphe 3 de l'article premier relatif aux agents publics régis par des statuts spécifiques, pour en faire des sujets actifs de la procédure de protection du

6 S. Gamonal C., La Eficacia Diagonal u Oblicua y los Estándares de Conducta en el Derecho del Trabajo, Santiago, Thomson Reuters, 2015, p. 123.

7 Rol 3.853-2017, 6 décembre 2018.

8 II s'agit d'un contrôle spécifique d'inconstitutionnalité, dans un cas ou un procès particulier. Art. 93 $n^{\circ} 6$ de la Constitution du Chili. 


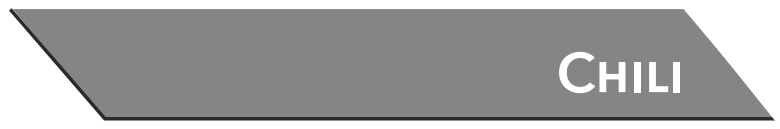

travail, dénature leur régime juridique et confie le soin aux tribunaux du travail d'examiner des cas qui ne relèvent pourtant pas de leur compétence légale expresse ».

Seuls quatre Ministres ont voté en faveur de l'application de la procédure de protection aux fonctionnaires et agents publics. Une minorité des membres de la Cour constitutionnelle s'est donc ralliée aux arguments de la Cour suprême, rappelant que l'interprétation des lois du travail doit être guidée par le principe in dubio pro operario, ajoutant que la Constitution ne fait pas de distinction entre les types de tribunaux, qu'ils soient civils ou du travail. D'autre part, le statut administratif ne prévoit aucune procédure de protection des droits fondamentaux. II existe donc bel et bien un vide juridique. Le Bureau du Contrôleur général de la République ne joue pas non plus ce rôle, comme le soutient le vote majoritaire, puisqu'il s'agit d'un recours administratif (non juridictionnel) pour vice de légalité. Ce vote dissident met en exergue l'importance des droits fondamentaux, piliers de relations de travail pleinement démocratiques, inviolables en toutes circonstances, que l'intéressé soit ou non un fonctionnaire.

\section{IV - CRITIQUES}

La défense des droits fondamentaux de tous est une priorité du droit chilien. En témoignent l'article 19 de la Constitution qui fait référence aux droits fondamentaux de toutes les personnes sans distinction, et le paragraphe 2 de l'article 5 de la même Constitution, qui fait référence au fait que l'État et ses organes doivent respecter et promouvoir les droits de I'homme en vigueur inscrits dans les traités internationaux ratifiés par le Chili. En matière de droits fondamentaux, le législateur tend lui-même à appliquer la réglementation du travail au secteur public. Cela a été le cas avec la protection de la maternité, l'interdiction du harcèlement sexuel et moral. Dans tous ces cas, la réglementation du Code du travail a été aussi appliquée au secteur public. En d'autres termes, s'il est vrai que les fonctionnaires disposent d'un statut spécial, le législateur a considéré qu'en matière de droits fondamentaux, la réglementation devait être identique pour les secteurs public et privé. Néanmoins, un grand vide juridique persiste s'agissant de la procédure de protection, dont le législateur n’a pas envisagé l'applicabilité au secteur public. Dès lors, la jurisprudence de la Cour suprême offre la possibilité d'appliquer la règle supplétive de l'article 1 paragraphe 3 du Code du travail et de fixer, dans la pratique, la défense des droits fondamentaux prévue par la Constitution. La décision de la Cour constitutionnelle a été critiquée et s'inscrit dans la tendance majoritaire d'extrême droite de ses membres? ${ }^{9}$. II n'est donc pas surprenant que la majorité de la Cour constitutionnelle chilienne cite la législation de la dictature comme si elle avait été une période de normalité démocratique et ne fait aucunement allusion aux traités relatifs aux droits de l'homme, pas plus qu'au paragraphe 2 de l'article 5 de la Constitution. L'action de la Cour constitutionnelle, enclave antidémocratique de Pinochet dans des domaines tels que la loi sur l'avortement ou la réforme du travail, a provoqué un vif débat sur la nécessité de la réformer rapidement et d'en limiter les pouvoirs ${ }^{10}$.

9 F. Atria, C. Salgado, «El TC como tercera cámara: la continuación de la política por otros medios », El Mostrador, 2015: https://www.elmostrador.cl/noticias/opinion/2015/03/09/el-tc-comotercera-camara-la-continuacion-de-la-politica-por-otros-medios/. Atria et Salgado y expliquent le fonctionnement de la Cour constitutionnelle du Chili en tant que « troisième Chambre » qui oppose son veto aux deux Chambres représentatives du peuple: le Sénat et la Chambre des Députés.

10 G. Gómez, "Constitucionalista Gastón Gómez y la posibilidad de reformar el TC: Hay un enorme consenso ", El Mostrador, 2019: https://www.elmostrador.cl/noticias/pais/2019/07/25/ constitucionalista-gaston-gomez-y-la-viabilidad-de-reformar-el-tc-hay-un-enorme-consenso/ 